\title{
OrthoWeb - web application for macro- and microevolutionary analysis of genes
}

\author{
Zakhar Mustafin \\ Kurchatov Genomics Center, \\ ICG SB RAS, Novosibirsk, Russia \\ mustafinzs@bionet.nsc.ru \\ Alexey Mukhin \\ Kurchatov Genomics Center, \\ ICG SB RAS, Novosibirsk, Russia \\ mukhin@bionet.nsc.ru
}

\author{
Dmitry Afonnikov \\ Kurchatov Genomics Center, \\ ICG SB RAS, Novosibirsk, Russia \\ NSU, Novosibirsk, Russia \\ ada@bionet.nsc.ru \\ Yury Matushkin \\ ICG SB RAS, Novosibirsk, Russia \\ mat@bionet.nsc.ru
}

\author{
Sergey Lashin \\ Kurchatov Genomics Center, \\ ICG SB RAS, Novosibirsk, Russia \\ NSU, Novosibirsk, Russia \\ lashin@bionet.nsc.ru
}

\begin{abstract}
Problems of macro- and microevolution remain key problems of biology. Phylostratigraphic analysis became one of the most popular methods for studying macroevolutionary characteristic of genes. It is based on estimation of the divergence time among orthologous genes. Along with methods of microevolutionary analysis (for example, $\mathrm{dN} / \mathrm{dS}$ ratio estimation), phylostratigraphic methods are increasingly included into the methodological arsenal of evolutionary bioinformatics. There are some software packages used for computational analysis of the evolution by using different phylostratigraphic and microevolutionary indices, but they require users to know some additional software or programming languages. Here we present OrthoWeb software to calculate such indices using web browser only.
\end{abstract}

Key words - evolution, phylostratigraphy, ortholog, gene network

\section{Motivation and Aim}

Problems of macro- and microevolution remain key problems of biology. Phylostratigraphic analysis proposed over a decade ago [1] became one of the most popular methods for studying macroevolutionary characteristic of genes. It is based on estimation of the divergence time among orthologous genes. Study of such genes may highlight the evolutionary age of genes and help to understand the evolution. Along with methods of microevolutionary analysis (for example, $\mathrm{dN} / \mathrm{dS}$ ratio estimation), phylostratigraphic methods are increasingly included into the methodological arsenal of evolutionary bioinformatics. There are some software packages (http://apps.cytoscape.org/apps/orthoscape,[2];

https://cran.r-project.org/web/packages/myTAI,[3];

https://github.com/arendsee/phylostratr,[4]), used for computational analysis of the evolution by using different phylostratigraphic and microevolutionary indices, but they require users to know some additional software (like Cytoscape [5]) or programming languages (like R). Here we present OrthoWeb software to calculate such indices using web browser only.

\section{Methods and Algorithms}

OrthoWeb is the software to calculate evolutionary analysis of genes, based on evolutionary analysis algorithms used in Orthoscape the software previously developed by our group. There are two groups of indices implemented in OrthoWeb.
PAI

PAI (Phylostratigraphic Age Index) aims at macroevolution analysis. It allows finding the "gene age" as a supposed stage of gene origin (the stage where the earliest duplication of ortholog can be found). The PAI analysis is based on comparison of the sequences of proteins, coded by original gene and its orthologous genes. There are two ways to compare genes and orthologs:

1) By using KEGG database (Kyoto Encyclopedia of Genes and Genomes, https://www.kegg.jp, [6]). There are two main thresholds allow us to check if genes are orthologous or not. The first one is the identity of amino acid sequences (as a part from 0 to 1 ) and the second is the SW-Score (the result of the Smith-Waterman alignment algorithm). The genes, which values of identity and SWScore in compare with original genes are higher than userdefined threshold are counted as orthologous genes. There is also featured threshold based on domains comparison, which can be used for more complex analysis. It is possible to specify the domains of proteins coded by analyzing genes.

2) By using BLAST (Basic Local Alignment Search Tool, https://blast.ncbi.nlm.nih.gov/Blast.cgi). This method based on uploading the sequence of gene into NCBI (National Center for Biotechnology Information) and analyzing it using BLAST. User can specify identity and score by the same way as in the method 1 .

Both methods return the list of orthologous genes and taxonomic lineages of organisms which genes are presented. The phylum most remote from the root of the taxonomic tree (Cellular Organisms) and common for gene and every ortholog represents the "gene age".

$D I$

DI (Divergence Index) aims at microevolution analysis. The index based on well-known ratio $\mathrm{dN} / \mathrm{dS}$, where $\mathrm{dN}$ shows the number of nonsynonymous substitutions and dS the number of synonymous substitutions. The ratio value above 1 indicates the evolution of the gene under positive Darwinian selection. The ratio close to 1 indicates that a gene evolves under neutral regime. The values of the ratio close to 0 indicate strong purifying selection acting on a gene.

DI is an average value of $\mathrm{dN} / \mathrm{dS}$ for gene and its orthologs. OrthoWeb allows to choose the degree of relationship between organisms but usually the closest 
organisms using in analysis (for example A.lyrata for A.thaliana or Hominidae's orthologs for H.sapiens).

To count dN/dS OrthoWeb uses PAML (Phylogenetic Analysis by Maximum Likelihood, http://abacus.gene.ucl.ac.uk/software/paml.html, [7]).

\section{Results}

OrthoWeb is available for analysis of gene sets. It is implemented using Java language. The server part is implemented using Spring framework (https://spring.io), the client part - Webix framework (https://webix.com).

Some results obtained with OrthoWeb were present on [8]. Currently is available by request to author.

\section{Acknowledgment}

The study is partially supported by RFBR, grant No. 20-04-00885 A, and Budget Project No. 0324-20190040-C-01.

\section{References}

[1] T. Domazet-Lošo, J. Brajković, and D. Tautz, "A phylostratigraphy approach to uncover the genomic history of major adaptations in metazoan lineages," Trends Genet., vol. 23, no. 11, pp. 533-539, Nov. 2007, doi: 10.1016/j.tig.2007.08.014.
[2] Z. S. Mustafin, S. A. Lashin, Y. G. Matushkin, K. V. Gunbin, and D. A. Afonnikov, "Orthoscape: a cytoscape application for grouping and visualization KEGG based gene networks by taxonomy and homology principles," BMC Bioinformatics, vol. 18, no. S1, pp. 1-9, 2017, doi: 10.1186/s12859-016-1427-5.

[3] H.-G. Drost, A. Gabel, J. Liu, M. Quint, and I. Grosse, "myTAI: evolutionary transcriptomics with R," Bioinformatics, vol. 34 , no. 9, pp. 1589-1590, May 2018, doi: 10.1093/bioinformatics/btx835

[4] Z. Arendsee, J. Li, U. Singh, A. Seetharam, K. Dorman, and E. S. Wurtele, "phylostratr: A framework for phylostratigraphy," bioRxiv, p. 360164, Jul. 2018, doi: 10.1101/360164.

[5] P. Shannon et al., "Cytoscape: A software Environment for integrated models of biomolecular interaction networks," Genome Res., vol. 13, no. 11, pp. 2498-2504, 2003, doi: $10.1101 /$ gr.1239303.

[6] M. Kanehisa, M. Furumichi, M. Tanabe, Y. Sato, and K. Morishima, "KEGG: New perspectives on genomes, pathways, diseases and drugs," Nucleic Acids Res., vol. 45, no. D1, pp. D353-D361, 2017, doi: 10.1093/nar/gkw1092.

[7] Z. Yang, "PAML 4: Phylogenetic analysis by maximum likelihood," Mol. Biol. Evol., vol. 24, no. 8, pp. 1586-1591, 2007, doi: $10.1093 / \mathrm{molbev} / \mathrm{msm} 088$.

[8] Z. S. Mustafin, V. I. Zamyatin, D. K. Konstantinov, A. V. Doroshkov, S. A. Lashin, and D. A. Afonnikov, "Phylostratigraphic Analysis Shows the Earliest Origination of the Abiotic Stress Associated Genes in A. thaliana," Genes (Basel)., vol. 10, no. 12, p. 963, Nov. 2019, doi: 10.3390/genes 10120963 